\title{
Novel epigenetic network biomarkers for early detection of esophageal cancer
}

\author{
Alok K. Maity', Timothy C. Stone ${ }^{2}$, Vanessa Ward², Amy P. Webster ${ }^{3}$, Zhen Yang ${ }^{4}$, Aine Hogan², Hazel McBain², \\ Margaraet Duku², Kai Man Alexander Ho², Paul Wolfson², David G. Graham²,5, SPIT Study Group, Stephan Beck ${ }^{6}$, \\ Andrew E. Teschendorff ${ }^{*}$ (i) and Laurence B. Lovat ${ }^{2,5^{*}}$
}

\begin{abstract}
Background: Early detection of esophageal cancer is critical to improve survival. Whilst studies have identified biomarkers, their interpretation and validity is often confounded by cell-type heterogeneity.

Results: Here we applied systems-epigenomic and cell-type deconvolution algorithms to a discovery set encompassing RNA-Seq and DNA methylation data from esophageal adenocarcinoma (EAC) patients and matched normal-adjacent tissue, in order to identify robust biomarkers, free from the confounding effect posed by cell-type heterogeneity. We identify 12 gene-modules that are epigenetically deregulated in EAC, and are able to validate all 12 modules in 4 independent EAC cohorts. We demonstrate that the epigenetic deregulation is present in the epithelial compartment of EAC-tissue. Using single-cell RNA-Seq data we show that one of these modules, a proto-cadherin module centered around CTNND2, is inactivated in Barrett's Esophagus, a precursor lesion to EAC. By measuring DNA methylation in saliva from EAC cases and controls, we identify a chemokine module centered around CCL20, whose methylation patterns in saliva correlate with EAC status.
\end{abstract}

Conclusions: Given our observations that a CCL20 chemokine network is overactivated in EAC tissue and saliva from EAC patients, and that in independent studies CCL20 has been found to be overactivated in EAC tissue infected with the bacterium F. nucleatum, a bacterium that normally inhabits the oral cavity, our results highlight the possibility of using DNAm measurements in saliva as a proxy for changes occurring in the esophageal epithelium. Both the CTNND2/CCL20 modules represent novel promising network biomarkers for EAC that merit further investigation.

Keywords: Esophageal adenocarcinoma, DNA methylation, Saliva, Barrett's esophagus, Single-cell RNA-Seq, Biological networks

\section{Background}

One of the most urgent needs in the clinical management of esophageal cancer is a reliable test for early detection of disease, which would help significantly towards improving what are currently dismal 5-year survival rates

\footnotetext{
*Correspondence: aeteschendorff21@outlook.com; l.lovat@ucl.ac.uk ${ }^{1}$ CAS Key Lab of Computational Biology, Shanghai Institute for Nutrition and Health, University of Chinese Academy of Sciences, Chinese Academy of Sciences, 320 Yue Yang Road, Shanghai 200031, China 2 Division of Surgery and Interventional Science, University College London, Gower Street, London WC1E 6BT, UK

Full list of author information is available at the end of the article
}

[1-3]. Given the low compliance to undergo endoscopy, an early detection test would ideally also be non-invasive and cheap enough to implement for routine screening. While many studies have identified promising epigenetic biomarkers for esophageal cancer [4-9], a key drawback hampering biological interpretation and successful validation is cell-type heterogeneity of analyzed tissues [10-13]. Cell-type heterogeneity refers to the presence of stromal cells, notably immune cells and fibroblasts, in addition to the resident epithelial cells of the tissue, with variations in the corresponding cell-type fractions generally accounting for most of the data-variance when 
analyzing DNA methylation or gene-expression [10]. Thus, it is important to adjust for such cell-type heterogeneity when inferring cancer biomarkers.

Here we aimed to identify robust biomarkers for esophageal adenocarcinoma (EAC) by using state-of-theart computational methodology to address the challenges posed by cell-type heterogeneity and multiple-testing. Specifically, we improve robustness and the false positive rate by not searching for individual differentially altered genes, but by searching for gene-modules that are jointly differentially methylated and differentially expressed, using our previously validated FEM (Functional Epigenetic Modules) algorithm to identify such modules in the context of a high quality protein-protein-interaction (PPI) network [14, 15]. A number of such integrative module detection algorithms have since emerged $[16,17]$, the rationale being that DNAm changes in disease often accompany gene expression changes that map to specific biological pathways and functional modules [15]. This systems-approach can therefore remove false positives and ensure the likelihood of discovering true positives, despite the crude nature of the underlying PPI networks. Because of the confounding effect posed by cell-type heterogeneity, we here extend FEM, by combining it with HEpiDISH [18], an algorithm designed to perform cell-type deconvolution of complex epithelial tissues. Briefly, we apply HEpiDISH to estimate total epithelial, immune and fibroblast fractions in the EAC samples from the TCGA cohort [19], and which are subsequently used to infer statistics of differential DNA methylation and mRNA expression reflecting associations with EAC that are not driven by underlying changes in cell-type composition. Following identification of FEM-modules associated with EAC, we perform extensive validation in independent EAC cohorts, and finally explore the potential utility of specific modules for early detection of EAC. We do this in the context of a single-cell RNA-Seq dataset comprising cells from Barrett's esophagus, a premalignant lesion that precedes EAC development. Our findings also lead us to explore the inferred gene-modules in the context of DNAm in saliva, an easily accessible tissue that contains a significant proportion of squamous epithelial cells [18, 20-22], which may serve as a suitable surrogate for recording DNAm changes in the cells that give rise to Barrett's esophagus and EAC.

\section{Results}

Identification of epigenetically deregulated gene-modules in EAC

The overall strategy to detect biomarkers for early detection of esophageal cancer was largely driven by power considerations and availability of appropriate datasets (Fig. 1). The underlying idea behind our strategy was to leverage the higher effect sizes and larger number of samples of EAC-tissue cohorts to identify EAC-biomarkers (Fig. 1a), and subsequently to filter these for potential relevance in early detection, either in preneoplastic esophageal tissue or in suitable surrogate tissues like saliva (Fig. 1b). In order to identify robust biomarkers associated with EAC, we combined a systems-epigenomics algorithm called FEM (Functional Epigenetic Modules) $[14,15]$ with a cell-type deconvolution algorithm called HEpiDISH [18], applying them both in an integrative fashion to the TCGA EAC cohort [19] (Fig. 1a). This cohort has genome-wide DNAm (12 normal-adjacent +50 EAC) and RNA-Seq (8 normal-adjacent +79 EAC) data available, allowing us to identify joint DNAm and mRNA expression changes in a predominantly stage T1-3 EACs cohort (approximately $20 \%$ of the tumors are $\mathrm{T} 1,34 \% \mathrm{~T} 2,41 \% \mathrm{~T} 3$ and only 5\% T4). By using FEM [14], we search for hotspots (gene-modules) of joint differential DNAm and mRNA expression between EAC and normal-adjacent tissue in the context of a protein-protein-interaction (PPI) network (Fig. 1a), a strategy that maximizes the chance of discovering true positive associations [23] and which we have previously and successfully applied to other cancer-types [15]. However, because normal and cancer tissue represent admixtures of esophageal epithelial cells with immune and other stromal cells (e.g. fibroblasts), we here extended the FEM algorithm to adjust for stromal heterogeneity when computing differential DNAm and mRNA expression statistics (Methods). In more detail, we used our HEpiDISH framework [18] to estimate total epithelial, total fibroblast and total immune cell fractions in each of the esophageal TCGA samples (Additional file 1: Fig. S1). SVD-analysis on the DNAm dataset revealed that the top principal component correlated most strongly with normal-cancer status (Additional file 1: Fig. S1). Cell-type fractions correlated strongly with lower ranked components but marginally also with PC1. Hence, when identifying differentially methylated genes (DMGs) and differential expressed genes (DEGs) between normal and cancer tissue, we used the estimated cell-type fractions as covariates in the linear regression models, so as to avoid confounding by potential changes in cell-type composition. Using this strategy, we identified a total of 12 gene-modules (Fig. 2, Additional file 1: Table S1, Additional file 1: Fig. S2), centered around 12 marker "seed" genes, which included CTNND2, CCL2O and NCAM1. For instance, the module around CTNND2 revealed widespread promoter hypermethylation and downregulation of many proto-cadherin genes, including CTNND2 itself (Fig. 2). In contrast, the CCL2O module was dominated by promoter hypomethylation and overexpression, suggesting activation of this chemokine-network in EAC (Fig. 2). For each module we 


\title{
Identification of network biomarkers for early detection of EAC
}

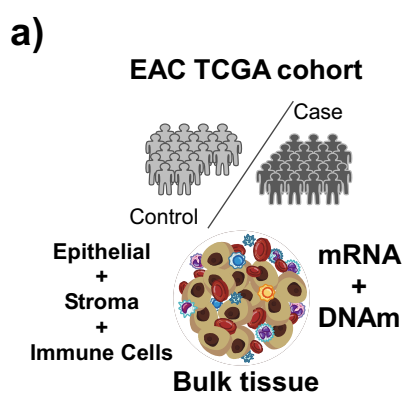

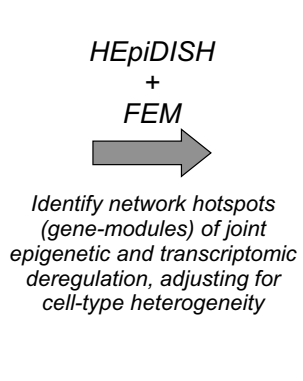

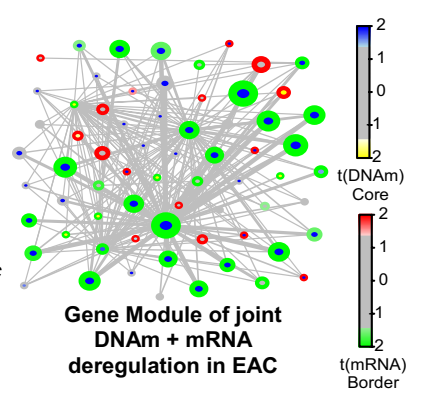

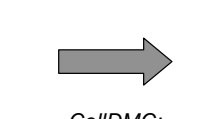

CellDMC:
gene modules altered
in epithelial compartment?

b)

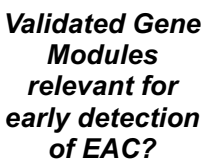

ScRNA-Seq data of Barrett's Esophagus (BE)
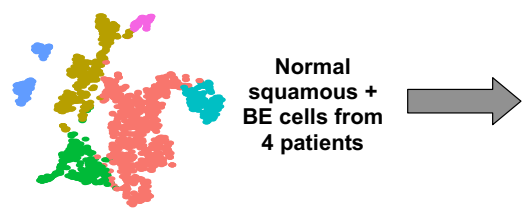

\section{CTNND2 protocadherin module}

\author{
independent \\ DNAm and mRNA \\ expression \\ EAC cohorts
}

Validation in

EAC saliva cohorts: Cohort-1 ( $n=163)$ Cohort-2 $(n=49)$

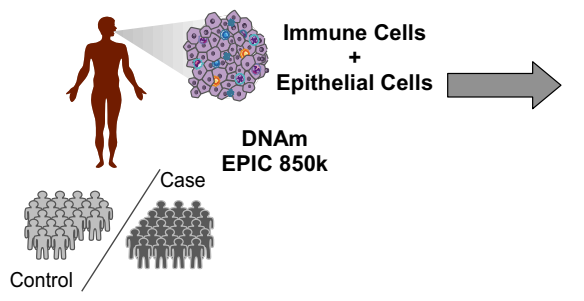

CCL20 chemokine module

Fig. 1 Identification of network biomarkers in EAC. a Using the TCGA DNA methylation and mRNA expression datasets for EAC, we identify gene-modules of joint epigenetic and expression deregulation in EAC compared to normal-adjacent tissue, using our Functional Epigenetic Modules (FEM) algorithm whilst adjusting for stromal heterogeneity. The latter is accomplished by estimating total epithelial, total immune cell and total fibroblast fractions in the TCGA samples using our HEpiDISH algorithm. FEM searches for gene-modules in the context of a PPI network. Subsequently, we apply our CellDMC algorithm to ascertain if the DNA methylation changes underlying the inferred gene modules are happening in the epithelial compartment of the tissue. Finally, we validate inferred modules in independent DNA methylation and mRNA expression EAC datasets. b Modules validating in (a) are then explored for their potential utility as early detection markers. This is done in two ways. In one case we analyse scRNA-Seq data from Barrett's and normal esophagus to explore if any modules are deregulated in Barrett's. In the second case, we explore if promising gene-modules exhibit variable DNA methylation patterns in saliva from cohorts containing both EAC and healthy subjects, where we also apply cell-type deconvolution methods to estimate epithelial fractions in saliva

computed a "FEM-activation score" (Methods) reflecting the degree of deregulation, which was generally speaking independent of tumor-stage (Additional file 1: Fig. S3). Despite some of the genes in these modules (e.g. CTNND2, CDH18, CCL20) displaying copy number variation (CNV) in esophageal cancer [19], we verified that the statistics of differential DNAm and mRNA expression for all gene module members were very robust upon adjustment for CNV-status (Additional file 1: Figs. S4, $\mathrm{S} 5)$, thus demonstrating that $\mathrm{CNV}$ is not a confounder.

\section{Validation of epigenetically deregulated gene-modules in independent cohorts}

To ascertain the biological significance of the 12 genemodules, we collated a total of 4 independent EAC cohorts, 2 profiling mRNA expression and 2 profiling DNAm
(Additional file 1: Table S2). For each module in each dataset, we computed a score (FEM-score), assessing the level of epigenetic or transcriptomic deregulation of each sample, and asked if the FEM-scores can discriminate normal from cancer tissue (Methods). In the case of DNAm we were able to validate all 12 modules with high statistical significance in both cohorts (Fig. 3). In the case of mRNA expression, the majority of modules were validated in at least one of the two cohorts (Additional file 1: Figs. S6-S9). Thus, this demonstrates that FEM has indeed identified bona-fide epigenetic and transcriptomic markers of EAC.

\section{Deregulation of FEM-modules occurs in the epithelial compartment}

Next, we wanted to assess in which cell-types the cancer-associated molecular alterations underlying 


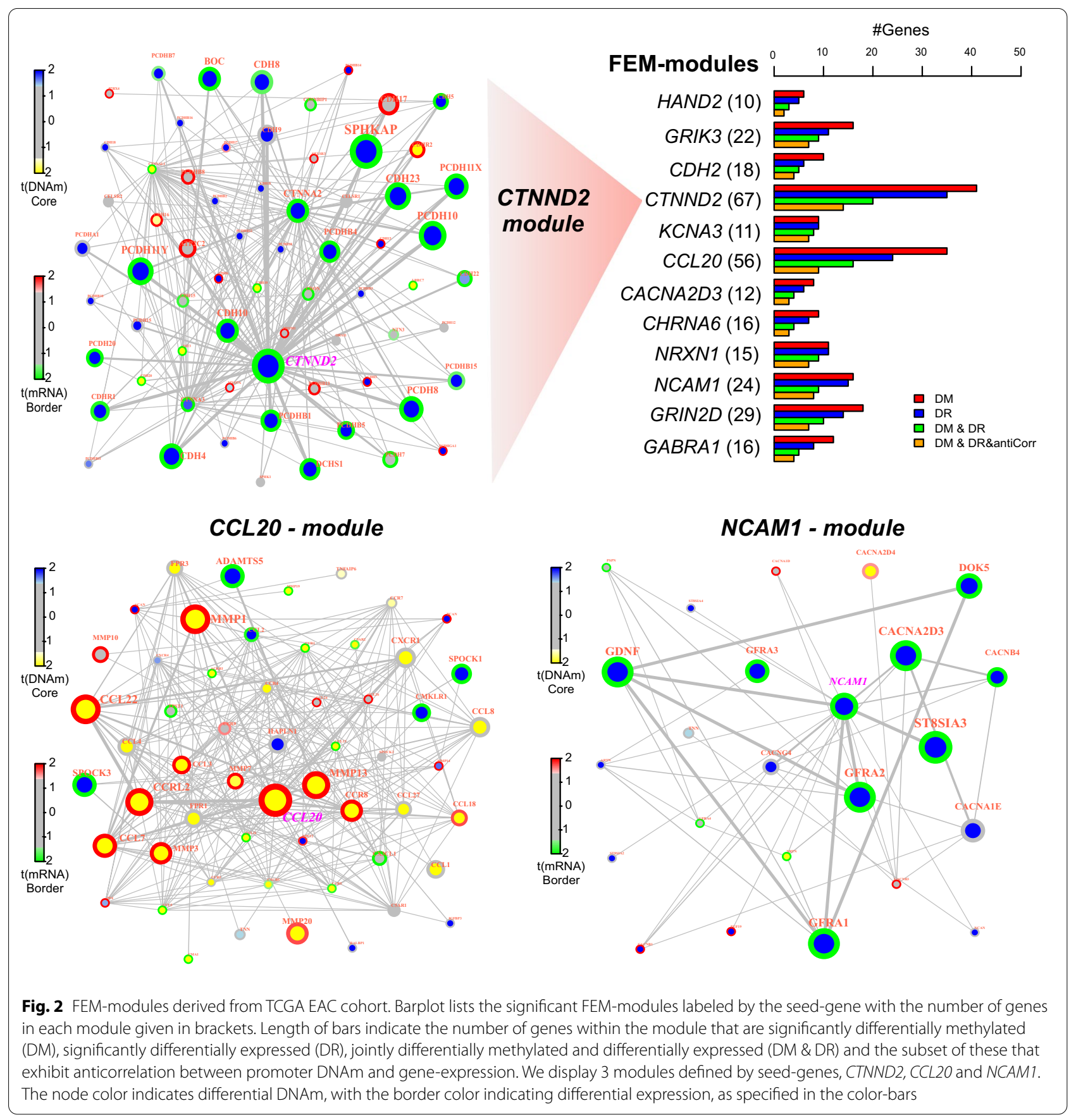

our FEM-modules occur. To this end, we applied CellDMC [24], an algorithm designed to detect cell-type specific DNAm changes to the discovery EAC DNAm dataset from the TCGA (Methods), in order to identify cell-type specific differentially methylated genes (cts-DMGs). For most FEM-modules, about half of the significantly differentially methylated genes within a module were predicted to be cts-DMGs, and by far these were mostly epithelial cts-DMGs (Fig. 4a). For instance, 24 of the 41 DMGs in the CTNND2-module were epithelial cts-DMGs, with only 1 being specific to the fibroblast compartment and none being specific to the immune-cell compartment. Only the CCL2O-module exhibited an equal number of cts-DMGs in the epithelial and fibroblast compartments. 


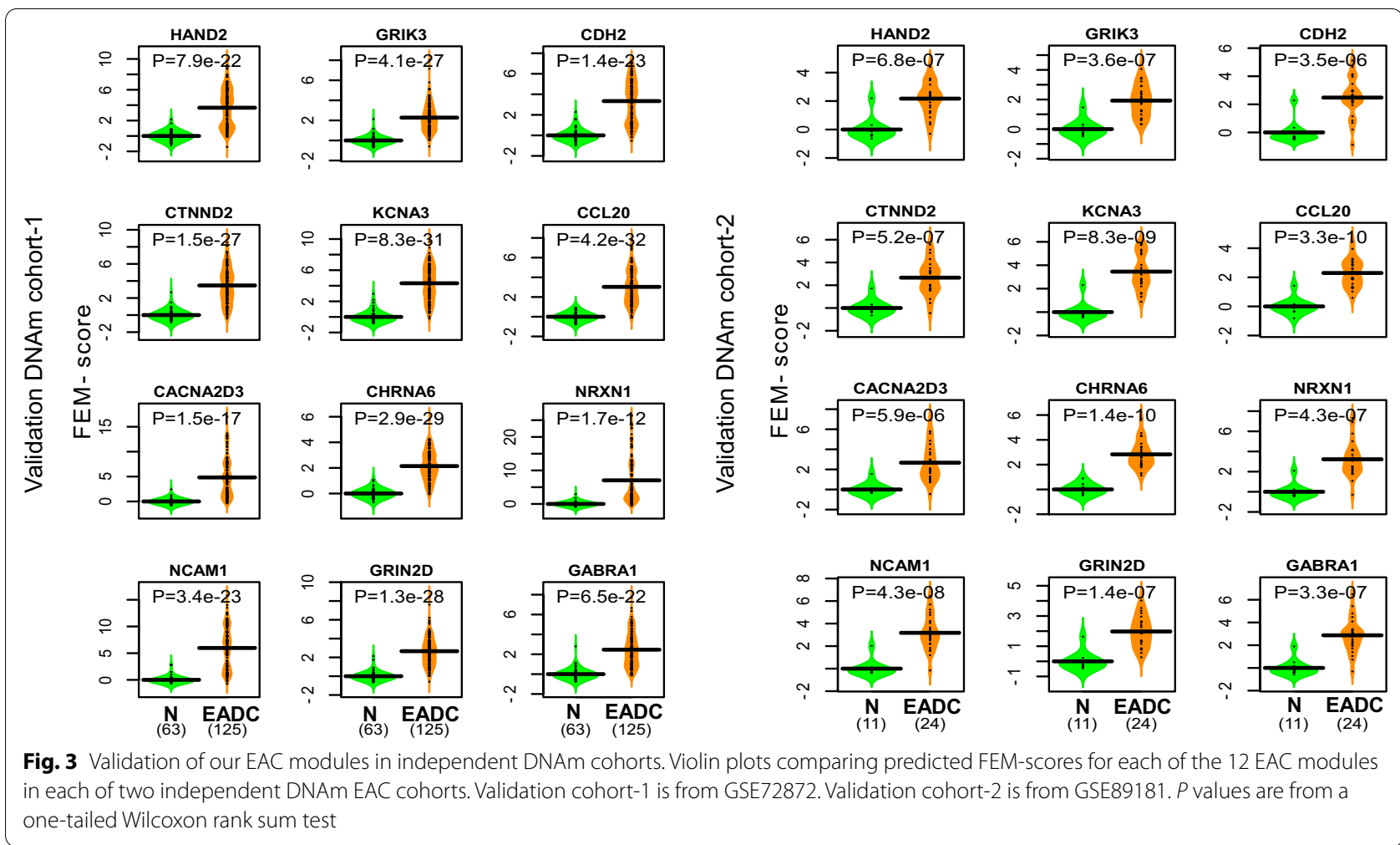

\section{The CTNND2 module is deregulated in the epithelial cells of Barrett's Esophagus}

To ascertain that our modules are capturing molecular changes in the epithelial cells of the esophagus, and to also explore the timing of the molecular alterations, we analyzed a Smart-Seq2 single-cell RNA-Seq dataset derived from 4 patients with Barrett's esophagus (BE) at each of 4 sites, including normal squamous epithelial cells as well as epithelial cells derived from BE, duodenum and stomach [25] (Methods). Indeed, we reasoned that some of our FEM-modules may already exhibit alterations in the epithelial cells from BE, which would make them more attractive for early detection strategies. Using the Seurat pipeline (Methods) we inferred a total of 6 clusters, with tissue/site and patient distributed unevenly across these clusters, but with the tissue/site dominating the source of variation and clustering (Fig. 4b). Next, we computed the FEM-score of our modules in each of the single cells (Methods). Unfortunately, due to the relatively high dropout rate, we could only compute the FEM-score for the CTNND2 module, since only for this module did we find enough gene members with variable expression across the 1038 cells (588 BE \& 450 normalsquamous). However, in each of the 4 patients the calculated FEM-score of the CTNND2 module was higher in the epithelial cells from BE compared to the adjacent normal squamous epithelium, and results were highly significant when merging the cells from all 4 patients together (Fig. 4c). Thus, these data indicate that the molecular alterations underlying the CTTND2-module is present in the esophageal epithelium as an early fielddefect in $\mathrm{BE}$.

\section{Association of CCL20 module with EAC in saliva}

Next, we assessed the 12 FEM-modules in two cohorts of saliva specimens (Cohorts 1 and 2) from subjects representing 4 different stages in EAC development, including normal $(N, n($ Cohort1 $)=65, n($ Cohort2 $)=5)$, nondysplastic BE (NDBE: $n(1)=33, n(2)=15)$, high grade dysplasia (HGD: $n(1)=14, n(2)=15)$ and cancer $(C: n(1)=51, n(2)=14)$ (Methods). Illumina DNAm profiles were generated for these cohorts using the EPIC platform that measures over $850,000 \mathrm{CpGs}$. The rationale for testing these modules in saliva at the DNAm level is that saliva contains a proportion of epithelial cells, likely derived from the buccal epithelium, which we posit may be informative of the DNAm changes seen in BE, HGD and EAC tissue.

To explore this, we first aimed to demonstrate that saliva contains a significant fraction of epithelial cells, and that these cells derive from the buccal epithelium of the oral cavity. We applied our HEpiDISH algorithm [18] to the two saliva cohorts in order to estimate the epithelial fraction. This confirmed that epithelial fractions were 


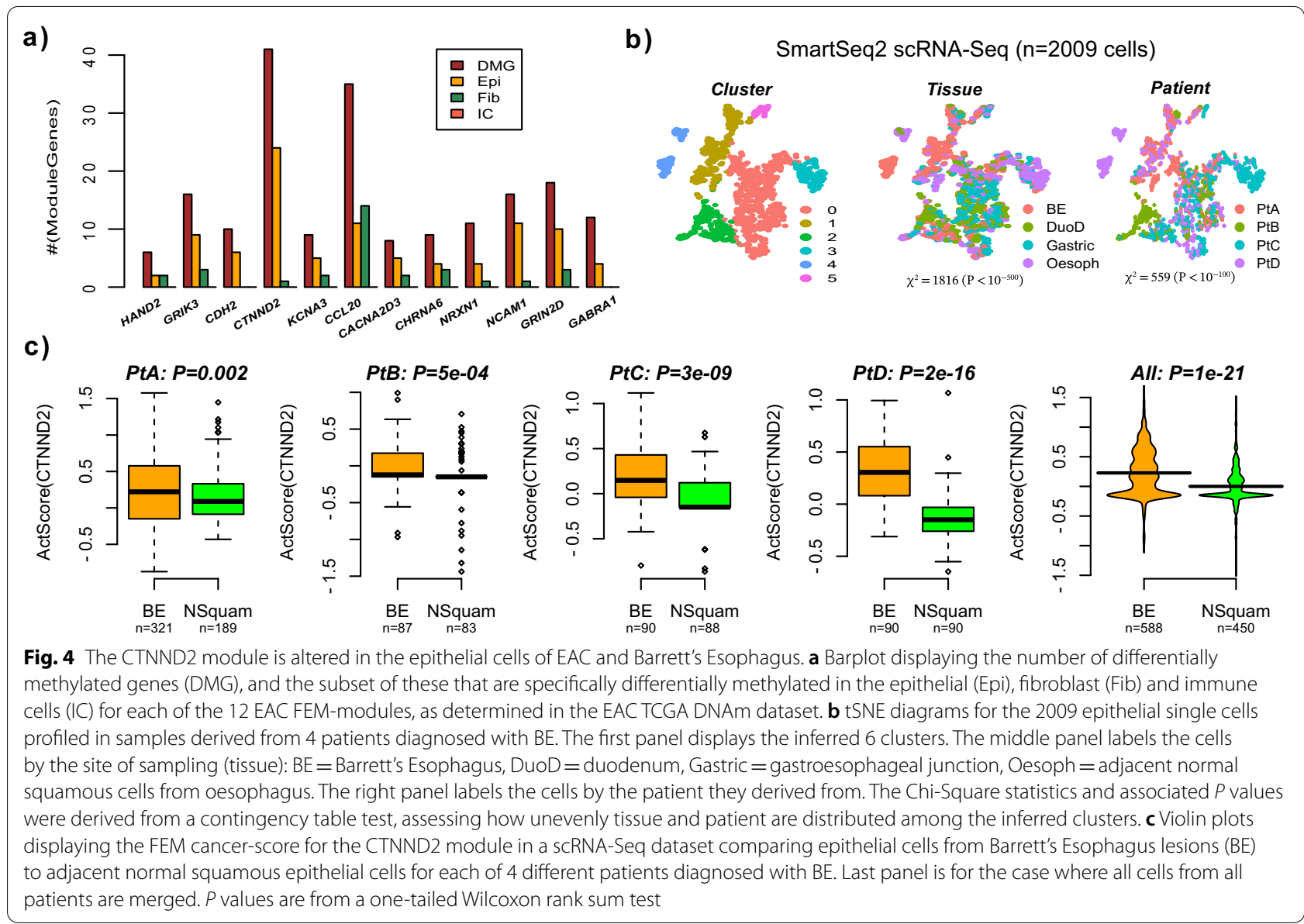

non-negligible, and interestingly, that they increased with cancer stage in both cohorts (Fig. 5a). To confirm the source of epithelial cells, we built a novel DNAm reference matrix with representative DNAm profiles for squamous buccal epithelium, EAC cell-lines and immune cells (Methods), and reapplied our HEpiDISH algorithm $[18,26]$ to infer corresponding cell-type fractions in our saliva samples. In both cohorts, this revealed an excellent correlation of the estimated buccal squamous epithelial content with the total epithelial fraction as determined with our previous DNAm reference matrix (Fig. 5b). In contrast, no correlations were observed between the total epithelial fraction and the fraction of esophageal adenocarcinoma cells (Fig. 5c). This confirms that the epithelial fraction in saliva derives from the adjacent squamous buccal epithelium. Finally, we computed the FEM-scores for all our modules in two saliva cohorts, which revealed a number of associations (Additional file 1: Figs. S10, S11). However, upon careful inspection most associations did not validate between Cohorts 1 and 2, with the exception of the CCL2O module, which did reveal a consistent increase with disease stage in both cohorts (Fig. 5d). Specifically, the CCL20 module displayed an $\mathrm{AUC}=0.65$
$(P=0.002)$ for discriminating normal from cancer in Cohort-1, and an AUC $=0.7(P=0.11)$ in Cohort-2, the non-significance in Cohort-2 attributable to the much smaller sample size of Cohort-2 (Fig. 5d, Additional file 1: Figs. S10, S11). Although these associations were partly driven by the increased epithelial fraction, the associations with EAC-status remained marginally significant under a linear regression model where we adjusted for the epithelial content (Cohort-1: $t=1.9, P=0.06$; Cohort-2: $t=1.8, P=0.09$ ). Thus, these data suggest that there are DNAm changes of the CCL2O module in saliva of EAC-cases that "mimick" those seen in EAC-tissue.

\section{Discussion}

Here we have identified two promising network biomarkers for EAC, one characterized by promoter hypermethylation and underexpression of a proto-cadherin gene module centered around CTNND2, and another module characterized by promoter hypomethylation and overexpression of a chemokine-network centered around CCL2O. Unlike previous studies, we identified these biomarkers by adjusting for variations in the 


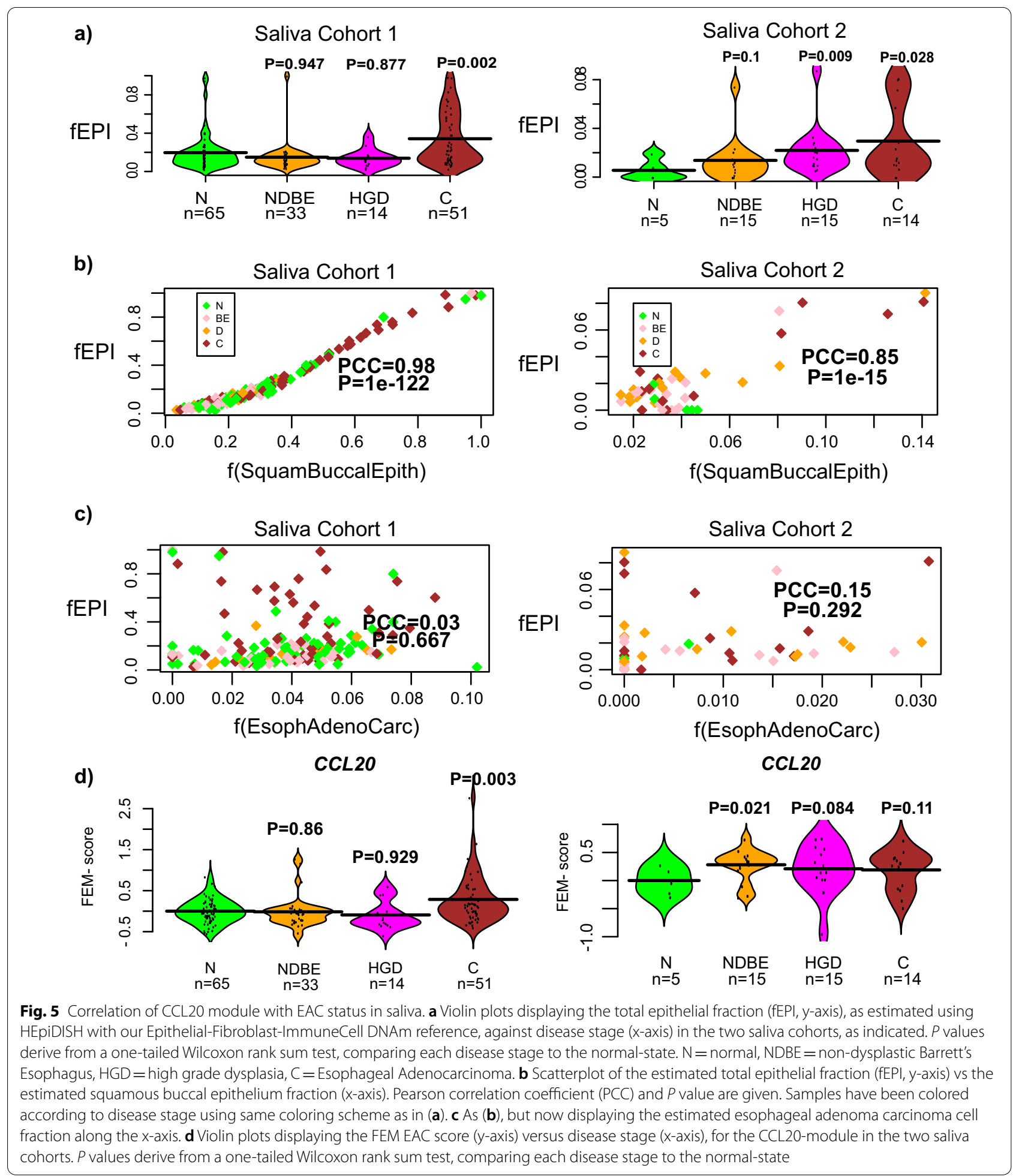

stromal compartment between normal and EAC-tissue, using state-of-the-art cell-type deconvolution methods, and furthermore demonstrating that the underlying DNAm chances are occurring primarily in the epithelial compartment of cells. In support of this we analysed scRNA-Seq data, demonstrating that the CTNND2module is aberrantly deregulated in the epithelial cells derived from Barrett's Esophagus in each of 4 separate BE 
patients. Thus, we have identified a potential field defect in $\mathrm{BE}$ that could be informative of disease progression. Admittedly, this association would be more relevant if demonstrated in BE patients with HGD, yet given that the CTNND2 module is not only deregulated in $\mathrm{BE}$ but also in EAC, it is highly plausible that the CTNND2 module would also be deregulated in intermediate stages such as BE + HGD lesions. Caution must be exercised however as our findings in BE are based on only 4 samples and results were derived from a scRNA-Seq data exhibiting over $90 \%$ sparsity. In any event, our data makes a strong case for scaling up scRNA-Seq studies to larger numbers of patients. While a number of previous studies have already indicated the potential importance of CTNND2/ WNT-signaling in EAC [27], a potentially more striking observation is that deletion of CTNND1 has been shown to lead to esophageal squamous cell carcinoma (ESCC) development $[28,29]$. Thus, it would appear that CTNND-genes play key tumor suppressor roles in both types of esophageal cancer, with their inactivation being mediated by potentially different molecular mechanisms (promoter hypermethylation in the case of CTNND2 and deletion in the case of CTNND1), which is possibly related to the different etiology of EAC vs ESCC.

CCL20/chemokine signaling has also been previously implicated in esophageal cancer [30]. Our observation that the CCL2O module appears to be overactivated in both EAC tissue as well as in saliva from EAC patients is striking, because an independent study has shown CCL2O to be strongly overexpressed in EAC-tissue displaying elevated levels of the bacterium F. nucleatum [31], a bacterium that also inhabits the oral cavity. It is therefore plausible that epigenetic changes associated with this bacterium in esophageal tissue could also be present in the buccal epithelial cells of the oral cavity. Of note, BE and EAC develop from metaplasia where squamous epithelial cells get replaced by columnar epithelium, thus the cell-of-origin of EAC is likely to be a basal progenitor squamous epithelial cell, similar to the cells in the buccal epithelium, thus providing further rationale as to why buccal epithelial cells may be an informative surrogate tissue of EAC. While we admit that this is hypothetical, our data clearly suggests an important future line of investigation: given that buccal swabs contain a much larger fraction of epithelial cells compared to saliva [18, 21 ], it would be interesting for future studies to jointly measure the microbiome and DNA methylome in such swabs, so as to explore the hypothesis that CCL2O (and DNAm alterations occurring elsewhere in the genome) is epigenetically deregulated as a function of F.nucleatum levels. Given the relative ease-of-access of buccal swabs, this could provide a promising avenue for developing non-invasive screening strategies for esophageal cancer.
There is also another reason why buccal swabs or saliva may be an appropriate tissue for assessing esophageal cancer risk. We have previously observed that DNAm changes that accrue in the buccal epithelium of smokers are very similar to those seen in a wide range of different epithelial cancer-types, including lung and esophagus [32]. It is likely that such common DNAm changes are driven by an increased cellular turnover and mitotic-rate associated with factors such as inflammation [33-35]. Inflammation could also be driven by bacterial infections, in addition to gastroesophageal reflux disease, the main risk factor for EAC. Thus, it is plausible that DNAm changes occurring in the buccal epithelium may record DNAm changes in other tissue-types that are also exposed to the same pathogen (F.nucleatum) or carcinogen (smoking).

Besides the sparsity and low number of clinical samples of the scRNA-Seq data, another major limitation of this study is the substantial inter-cohort and inter-individual variation of the epithelial fraction in saliva. These differences between individuals and cohorts likely stem from differences in sample collection and processing, but may also be influenced by confounders such as alcohol consumption, gingivitis or other medical conditions of the oral cavity. Of note, such high inter-individual variability has also been observed in buccal swabs $[18,20]$. Thus, moving forward, future work will need to understand the sources of inter-individual variability in epithelial fractions within saliva and buccal swabs.

\section{Conclusions}

In summary, by using innovative computational methods, we have here identified CTNND2 and CCL2O modules that represent two promising novel network biomarkers for early detection of EAC. We propose future studies to fully explore the clinical significance of these modules.

\section{Methods \\ Normalization and preprocessing of tissue EAC datasets TCGA EAC DNAm, mRNA expression and CNV dataset}

We used the DNAm, mRNA expression and CNV datasets from the TCGA [19]. These datasets were normalized as described by us previously [36, 37]. Briefly, in the case of DNAm data, missing values in the level-3 data were imputed using impute.knn [38] with $k=5$ and an imputation threshold of $30 \%$ (i.e. only probes with less than $30 \%$ missing values imputed, rest of probes were removed), followed by BMIQ normalization [39]. There were 12 normal-adjacent +50 EAC samples measured with Illumina $450 \mathrm{k}$ beadarrays, and 8 normal-adjacent +79 EAC samples measured with RNA-Seq. 


\section{Validation bulk tissue DNAm datasets}

- Krause et al. [40] is an Illumina $450 \mathrm{k}$ DNAm dataset comprising genome-wide DNA methylation profiles for 250 samples including $125 \mathrm{EAC}$ and 64 normal adjacent squamous samples. Data is available from GEO (http://www.ncbi.nlm.nih.gov/geo/ under accession number GSE72872). Raw idat files were processed with minfi [41]. Probes with SNPs or with more than $25 \%$ missing values were removed. Rest of probes were imputed with impute.knn [38] with $\mathrm{k}=5$. Type -2 probe bias was corrected using BMIQ [39]. One sample was removed due to low quality as assessed using BMIQ, resulting in 63 normal adjacent and 125 EAC samples.

- Kaz et al. [42] is an Illumina $450 \mathrm{k}$ DNAm dataset comprising genome-wide DNA methylation profile of 127 esophageal samples, including 24 EAC and 11 normal adjacent squamous. Data is available from GEO (http://www.ncbi.nlm.nih.gov/geo/ under accession number GSE89181). Raw idat files were processed as described for Krause et al.

\section{Validation bulk tissue gene expression datasets}

- Krause et al. [40] performed Illumina gene expression (HumanHT-12 V4.0 bEAChip) profiling of 65 esophageal samples (48 EAC, 17 normal adjacent squamous). We downloaded the provided log-normalized dataset from GEO (http://www.ncbi.nlm.nih.gov/ geo/ under accession number GSE72874) and performed inter-sample normalization using quantile normalization with limma R package [43].

- Lu et al. [44] used Affymetrix Human Gene 1.0 ST Arrays to profile 10 normal adjacent squamous and 12 EAC. Raw data was downloaded from GEO (http://www.ncbi.nlm.nih.gov/geo/under accession number GSE92396). We successively applied intrasample and inter-sample normalization in the dataset using affy and limma R packages, respectively.

\section{scRNA-Seq dataset of Barrett's Esophagus (BE)}

We analysed a scRNA-Seq Smart-Seq2 dataset from Owen et al. [25] which profiled BE specimens as well as normaladjacent tissue for a number of different patients. We downloaded the gene-count matrix from the website provided with the publication, and processed it with the Seurat pipeline [45]. We selected cells with at least 200 expressed genes, and selected genes expressed in at least 3 cells. Counts were then log-normalized using a scale factor of $10^{4}$. In total we obtained 2009 cells from 4 different patients and from 4 distinct tissues: normal esophagus $(n=450)$, duodenum $(n=349)$, gastric $(n=622)$ and BE $(n=588)$. To assess the sources of variability in this dataset we ran the standard Seurat pipeline, including variable feature selection (with variance stabilization), PCA, graph-based clustering and tSNE visualization. Graph-based clustering was done over 8 components (inferred with ElbowPlot function) and at a resolution of 0.1 , which resulted in 6 clusters.

\section{Inference of FEM-modules from TCGA EAC dataset}

To identify epigenetically deregulated gene-modules in EAC, we applied our Functional Epigenetic Module (FEM) algorithm [14]. Briefly, this approach integrates DNA methylation with gene expression data in the context of a protein-protein-interaction (PPI) network to identify hotspots (gene-modules) where there is substantial coordinated DNAm and gene expression changes. The algorithm consists of three steps: (1) quantify t-statistics of differential DNAm (DM) and gene expression (DE) between adjacent normal and EAC for each gene in the PPI network, (2) the edges of the network are weighted according to an integrated statistic for each gene defining the edge, where the integrated statistic per gene is obtained from the corresponding differential DNAm and differential expression statistics, and (3) inference of hotspots as subnetworks of particularly high modularity, i.e. subgraphs where the average weighted edge density is high compared to the rest of the network. The PPI network consists of 11,751 genes annotated to NCBI Entrez identifiers and is derived from the Pathway Commons resource [46, 47]. In step-(1), in order to arrive at a single statistic for DM, we summarize DNAm for each gene as the average over TSS200 probes (i.e. probes within $200 \mathrm{bp}$ of the TSS). If such probes are not available, we use $1^{\text {st }}$ Exon probes instead, and if also not available then we use the average over TS1500 probes. This strategy is motivated by the fact that for these regions, there is generally an inverse relation between DNAm and gene expression, and the algorithm seeks to identify gene modules where this pattern is observed frequently. To derive the statistics for DM and DE we then use the limma R-package [43, 48, 49]. In step-(2), the integrated statistic per gene is then constructed as

$$
\begin{aligned}
t_{g}^{(I)}= & \left\{H\left(t_{g}^{(D M)}\right) H\left(-t_{g}^{(D E)}\right)\right. \\
& \left.+H\left(-t_{g}^{(D M)}\right) H\left(t_{g}^{(D E)}\right)\right\}\left|t_{g}^{(D M)}-t_{g}^{(D E)}\right|
\end{aligned}
$$

where $H(x)$ is a Heaviside function, $H(x)=1$ if $x>0$ and $H(x)=0$ if $\mathrm{x}<0$. If genes $g$ and $h$ are connected in the PPI network, we then assign the edge weight by taking the average of the corresponding integrated statistics of the genes, i.e. 


$$
w_{g h}=0.5 *\left(t_{g}^{(I)}+t_{h}^{(I)}\right)
$$

To infer the modules ("FEM-modules") in step-3, we then use a local greedy adaptation of a powerful spinglass algorithm $[23,50]$. The spin-glass algorithm tries to minimize the following Hamiltonian energy function

$$
H(\{s\})=-\sum_{g \neq h}\left\{w_{g h}-\gamma p_{g h}\right\} \delta\left(s_{g}, s_{h}\right)
$$

where $s_{g}$ is the spin-state (i.e., module) the gene $g$ belongs to, $\delta(x, y)=1$ iff $x=y$ and 0 otherwise, and $p_{g h} \sim w_{g} . w_{h}$. is the null probability (once normalized) with $w_{g}$. denoting the weighted degree of gene $g$. In the local greedy version we try to grow modules around a number of "seed-genes" defined as the highest ranked genes by the integrated statistic $t_{g}^{(I)}$. We choose on the order of 100 seed genes, to ensure that most of the network is explored. In previous studies we have found that this number of seed genes works well and already leads to redundant modules. The spin-glass parameter $\gamma$ was chosen to be 0.5 , as this parameter choice typically leads to modules in the size range of $10-100$, which is the optimal size-range as shown by us previously [23]. The statistical significance of the modules is determined in two complementary ways: a module is grown from a given seed-gene in a deterministic fashion by adding the neighboring gene that minimizes the Hamiltonian energy and this process is continued until no further additions can decrease the energy function. This assesses the significance of the edge-weight density of the module in relation to the rest of the network and is strongly influenced by the topology of the network. As a second topology-independent test, we assess statistical significance of the inferred modules using a Monte Carlo (MC) randomization procedure (1000 Monte-Carlo runs), where we randomize the statistics over the network (thus keeping the topology fixed), recomputing modularity scores for each module and subsequently comparing the observed modularity to that of this empirically generated null distribution. The final FEM-modules are those with a $P<0.05$.

\section{Computation of FEM-score in validation tissue EAC datasets}

In the case of the DNAm validation sets, we first summarized the Illumina $850 \mathrm{k} / 450 \mathrm{k}$ DNAm values to the promoters of the genes involved in a given module. This is done following our previously validated procedure [14]. This procedure averages the DNAm values of CpG probes mapping to within $200 \mathrm{bp}$ of the TSS of each gene. If no probes map to within $200 \mathrm{bp}$, we use probes mapping to the 1st Exon, and if not available, we resort to probes mapping $1.5 \mathrm{~kb}$ upstream of the TSS. Subsequently, we select the genes in the module that were significantly differentially methylated in the original EAC TCGA cohort. For each of these genes, we then z-score normalize their DNAm profile over all samples within the given validation cohort, using the mean and standard deviation as estimated over the samples from normal/healthy individuals. That is, if $x_{g s}$ denotes the DNAm value for gene $g$ in sample $s$, we compute

$$
z_{g s}=\frac{x_{g s}-\mu_{g N}}{\sigma_{g N}}
$$

where $\mu_{g N}, \sigma_{g N}$ denote the mean and standard deviation DNAm of gene $g$ across the normal samples. The FEMscore for module $m$ in sample $s$ is then obtained as

$$
\text { FEMscore }_{m s}=\frac{1}{|m|} \sum_{g \in m} \operatorname{Sign}\left(t_{g}\right) z_{g s}
$$

where $|m|$ is the number of significantly differentially methylated genes in the module (as determined by the discovery TCGA EAC dataset) and $\operatorname{Sign}\left(t_{g}\right)$ is the sign $(+1 /-1)$ of the corresponding t-statistic of differential DNAm from the TCGA EAC cohort. Thus, the FEMscore assesses whether the deviation in DNAm relative to the normal state is consistent with the pattern observed in the TCGA EAC cohort, with a higher FEMscore in cancer patients indicating a coordinated epigenetic deregulation consistent with that seen in the discovery TCGA set.

In the case of the gene-expression datasets, the FEMscore of the module was computed by z-score normalizing the expression values of the module genes, using the mean and standard deviation of the gene over the normal samples, in direct analogy to the DNAm case. Here, only genes that were significantly differentially expressed in the discovery TCGA EAC cohort are used. Given the $\mathrm{z}$-scores, the FEMscore is then obtained as the weighted average over all significant module genes, with the weight being +1 if the gene is overexpressed in cancer relative to normal according to the TCGA dataset, and -1 if underexpressed.

\section{Computation of FEM-score in scRNA-Seq data of BE}

Here we selected cells from normal esophagus $(n=450)$ and $\mathrm{BE}(n=588)$, and ran the above analysis to compute FEM-scores in each single cell, using the normal cells to define the z-score transformation. FEM-scores could only be reliably computed for the CTNND2-module: the number of significantly differentially expressed genes (from the TCGA EAC cohort) in each module that were also variable in the scRNA-Seq dataset was less than 5 for all modules except CTNND2 for which the number of variable genes was 11. FEM-scores were then compared 
between the normal and BE cells using Wilcoxon rank sum tests, stratified by patient.

\section{Computation of FEM-score in TCGA ESCA data}

The procedure for computing the FEM-score in the TCGA data was slightly different from the previously described one, because for the TCGA we have both DNAm and RNA-Seq data. Briefly, the FEM-score for module $m$ in sample $s$ was computed as

$$
\text { FEMscore }_{m s}=\frac{1}{|m|} \sum_{g \in m}\left|z_{g s}^{(M)}-z_{g s}^{(R)}\right|
$$

where $|m|$ is the number of significantly and consistently differentially methylated and differentially expressed genes in the module (as determined in the TCGA dataset itself), and where $z_{g s}^{(M)}$ and $z_{g s}^{(R)}$ are the corresponding z-scores for the DNAm and RNA-Seq datasets. By consistently differentially methylated and differentially expressed we mean that the pattern is anti-correlative (i.e., promoter hypermethylation and underexpression, or promoter hypomethylation and overexpression). The z-scores were computed as described previously, with the exception of the scores for the DNAm data where we added a constant offset term to the standard deviation, since the number of normal-adjacent samples with both DNAm and mRNA data is small $(n=6)$ and this can lead to spurious low variances and inflated scores in the DNAm-case. Specifically, for the DNAm-data we defined

$$
z_{g s}^{(M)}=\frac{x_{g s}-\mu_{g N}}{\sigma_{g N}+\gamma}
$$

where $\gamma$ was determined by requiring that the standard deviation of the $z$-scores for DNAm, as evaluated genome-wide over all genes in the experiment, equals the standard deviation of the $z$-scores for RNA-Seq data.

\section{Saliva sample collection}

Saliva was collected from two groups of patients: the primary "training" cohort of 192 patients was collected through the SPIT Study (Saliva to predict risk of disease using transcriptomics and epigenetics, ISRCTN11921553) at 15 hospitals in the United Kingdom. This study was ethically approved by the West Midlands-Coventry and Warwickshire Research Ethics Committee (REC Reference No: 17/WM/0079). The validation cohort of 49 patients were collected through the BOOST Study (Barrett's oesophagus surveillance with optical biopsy using spectroscopy and enhanced endoscopic imaging to target high-risk lesions, ISRCTN58235785) at a single hospital site (UCLH). This study was ethically approved by the
London-Dulwich Research Ethics Committee (REC Reference No: 08/H0808/8). All participants gave written consent.

Patients were instructed to fast for a minimum of one hour and then to spit repeatedly into a saliva DNA collection device to a total of $2 \mathrm{~mL}$. The primary cohort used Oragene DNA OG-500 (DNAGenotek, Ottawa, Canada) and the validation cohort used SimplOFy ${ }^{\mathrm{TM}}$ (Oasis Diagnostics ${ }^{\circledR}$ Corporation, Vancouver, Canada). Saliva was sent to the central laboratory at UCL and stored at $-80{ }^{\circ} \mathrm{C}$ until extraction.

DNA Extraction was carried out using the Zymo Quick-DNA $^{\mathrm{TM}}$ Miniprep Plus Kit (Zymo Research, Irvine CA, USA). DNA quality was assessed using the Agilent Bioanalyser (Agilent Inc, Santa Clara, CA, USA). Only samples with a $260 / 280 \mathrm{~nm}$ ratio of $>1.5$ were used. Bisulphite conversion was undertaken using the Zymo EZ-96 DNA methylation kit (Zymo Research, Irvine CA, USA) and samples containing 500 ng DNA were then DNAm profiled using the Illumina Infinium HumanMethylationEPIC BeadChip (Illumina San Diego, CA, USA).

\section{Analysis of saliva DNAm datasets}

Raw signal intensities were processed from idat files through a standard pipeline that we have extensively validated [18]. Briefly, we processed idat files with the minfi R-package with no background correction and using the Illumina definition of beta-values. Probes with $P$ values of detection $<0.05$ were declared reliable measurements, the rest being set to NA. We removed cross-reactive probes, probes with more than 4 SNPs, probes with a SNP at the interrogated $\mathrm{CpG}$, and probes with less than 90\% coverage. Remaining NAs were imputed with the impute R-package using impute.knn function with $k=5$ [38]. Type-2 probe bias was corrected using our BMIQ algorithm [39]. The final beta-valued data matrix for the discovery cohort contained 689,033 probes and 163 samples, of which 65 were from healthy controls, 33 from patients diagnosed with non-dysplastic Barrett's Esophagus (NDBE), 14 with high-grade dysplasia (HGD) and 51 with EAC. In the case of the validation cohort, a total of 49 samples passed QC, of which 5 were normal, 15 NDBE, 15 HGD and 14 EAC.

\section{Computation of FEM-score in saliva EAC DNAm datasets}

We applied the same procedure as for the tissue DNAm validation sets described above. Here, the normal samples used for performing the $\mathrm{z}$-score transformation are the saliva samples from the age-matched healthy controls. 


\section{Estimation of cell-type fractions in tissue and saliva DNAm datasets}

In the case of the TCGA EAC cohort we estimated celltype fractions from the Illumina $450 \mathrm{k}$ DNAm data with our HEpiDISH algorithm $[13,18]$. Briefly, this uses a DNAm reference matrix defined over 3 broad cell-types (total epithelial, total fibroblast and total immune-cell), in conjunction with Robust Partial Correlations (RPC) to yield estimates for the total epithelial, total fibroblast and total immune-cell fractions in each of the TCGA samples. In the case of the saliva DNAm datasets, the same procedure was applied to obtain total epithelial and total immune-cells fractions (the fibroblast fraction in saliva is negligible, which was also confirmed by HEpiDISH).

To assess the source of epithelial cells in saliva, we built a separate DNAm reference matrix defined over 3 main cell-types: squamous epithelial cells, epithelial cells representative of EAC cell-lines, and a generic immune-cell. In detail, the Illumina $450 \mathrm{k}$ DNAm data from 48 purified immune-cells $(6 \times 8$ cell-types $)$ was derived from Reinius et al. [51]. From Iorio et al. [52] we obtained Illumina $450 \mathrm{k}$ DNAm profiles for a total of 7 EAC cell-lines (ESO26, ESO51, FLO-1, KYAE-1, OACM5-1, OACP4C an SK-GT-4). For the squamous epithelial cells we first identified buccal swabs with over 95\% purity, as determined with our HEpiDISH algorithm with the DNAm reference matrix defined above, and as applied to our large buccal swab $450 \mathrm{k}$ DNAm dataset of 790 buccal swabs [32]. We only considered neversmokers to avoid DNAm changes induced by smoking. This resulted in 10 buccal swab samples of high epithelial purity, which we took as representative for putative squamous epithelial cells in saliva. To select the features (CpGs) defining our DNAm reference matrix, we performed differential DNAm analysis between the 48 IC, 7 EAC cell-line and 10 squamous epithelial cell samples using the limma empirical Bayes framework $[49,53]$. Specifically, we performed 3 pairwise comparisons (IC vs. EAC + SqEpi), (SqEpi vs IC + EAC) and (EAC vs. IC + SqEpi). In all cases, we selected features with an $\mathrm{FDR}<0.05$ and a difference in mean DNAm greater than 0.9 , except for the second comparison where we relaxed the threshold a little to 0.875 . This ensured similar numbers of differentially methylated CpGs (DMCs): 136, 123 and 153 for the 3 comparisons, respectively. This resulted in a DNAm reference matrix defined over 412 unique CpGs and 3 cell-types (total IC, squamous epithelial, EAC). To estimate cell-type fractions in saliva, we then applied our HEpiDISH/RPC algorithm with this DNAm reference matrix.

\section{Abbreviations}

EAC: Esophageal adenocarcinoma; BE: Barrett's esophagus; DMG: Differentially methylated gene; DMC: Differentially methylated CpG; FEM: Functional epigenetic modules; cts-DMG: Cell-type specific differentially methylated gene; FDR: False discovery rate; DNAm: DNA methylation; NDBE: Non-dysplastic Barrett's esophagus; HGD: High-grade dysplasia; PPI: Protein-protein-interaction; TCGA: The Cancer Genome Atlas; CNV: Copy-number variation.

\section{Supplementary Information}

The online version contains supplementary material available at https://doi. org/10.1186/s13148-022-01243-5.

Additional file 1. Contains all Supplementary Figures and Supplementary Tables.

\section{Acknowledgements}

The results shown here are in part based upon data generated by the TCGA Research Network (http://cancergenome.nih.gov/) to which we are very grateful.

\section{Authors' contributions}

The idea of using DNA methylation measurements in saliva for esophageal cancer prediction was conceived by LL. The bioinformatic strategy was conceived by AET. AKM, TS and AET performed statistical analysis with input from YZ. SB helped with the experimental protocols of the studies. All other co-authors contributed to the data generation process. All authors read and approved the final manuscript.

\section{Funding}

AET was supported by NSFC (National Science Foundation of China) Grants 31771464, 31970632 and by a Royal Society Newton Advanced Fellowship (NAF project number: 522438, NAF Award Number: 164914). LBL is supported by the National Institute for Health Research University College London Hospitals Biomedical Research Centre and the CRUK Experimental Cancer Medicine Centre at UCL as well as the Wellcome/EPSRC Centre for Interventional and Surgical Sciences (WEISS) at UCL; [203145Z/16/Z]

\section{Availability of data and materials}

The discovery EAC tissue mRNA and DNAm datasets are derived from the TCGA and are freely available from https://portal.gdc.cancer.gov/. The 4 EAC datasets used for validation are available from GEO http://www.ncbi.nlm. nih.gov/geo/ under Accession Numbers GSE72874, GSE92396, GSE72872, GSE89181. The scRNA-Seq dataset from BE as well as the other Illumina $450 \mathrm{k}$ DNAm datasets used here are all publicly available as specified in the respective Methods section and published references. The saliva Illumina DNA methylation datasets are only available on a collaborative basis by submitting a request to Prof. Laurence Lovat (I.lovat@ucl.ac.uk).

\section{Declarations}

\section{Ethics approval and consent to participate}

The SPIT Study (Saliva to predict risk of disease using transcriptomics and epigenetics, ISRCTN11921553) was ethically approved by the West Midlands-Coventry and Warwickshire Research Ethics Committee (REC Reference No: 17/ WM/0079). The BOOST Study (Barrett's oesophagus surveillance with optical biopsy using spectroscopy and enhanced endoscopic imaging to target highrisk lesions, ISRCTN58235785) was ethically approved by the London-Dulwich Research Ethics Committee (REC Reference No: 08/H0808/8). All participants gave written consent.

\section{Consent for publication}

Not applicable.

\section{Competing interests}

The authors declare no competing interests. 


\section{Author details}

${ }^{1}$ CAS Key Lab of Computational Biology, Shanghai Institute for Nutrition and Health, University of Chinese Academy of Sciences, Chinese Academy of Sciences, 320 Yue Yang Road, Shanghai 200031, China. ${ }^{2}$ Division of Surgery and Interventional Science, University College London, Gower Street, London WC1E 6BT, UK. ${ }^{3}$ University of Exeter Medical School, University of Exeter, Exeter, UK. ${ }^{4}$ Key Laboratory of Medical Epigenetics and Metabolism, Institutes of Biomedical Sciences, Fudan University, Shanghai 200032, China. ${ }^{5}$ Division of GI Services, University College London Hospitals NHS Foundation Trust, 235 Euston Road, London NW1 2BU, UK. ${ }^{6}$ UCL Cancer Institute, University College London, Gower Street, London WC1E 6BT, UK.

\section{Received: 28 July 2021 Accepted: 4 February 2022}

\section{Published online: 14 February 2022}

\section{References}

1. Enzinger PC, Mayer RJ. Esophageal cancer. N Engl J Med. 2003;349:2241-52.

2. Ross-Innes CS, Chettouh H, Achilleos A, Galeano-Dalmau N, DebiramBeecham I, MacRae S, Fessas P, Walker E, Varghese S, Evan T, et al. Risk stratification of Barrett's oesophagus using a non-endoscopic sampling method coupled with a biomarker panel: a cohort study. Lancet Gastroenterol Hepatol. 2017;2:23-31.

3. Adil Butt M, Pye H, Haidry RJ, Oukrif D, Khan SU, Puccio I, Gandy M, Reinert HW, Bloom E, Rashid M, et al. Upregulation of mucin glycoprotein MUC1 in the progression to esophageal adenocarcinoma and therapeutic potential with a targeted photoactive antibody-drug conjugate. Oncotarget. 2017;8:25080-96.

4. Kaz AM, Grady WM. Epigenetic biomarkers in esophageal cancer. Cancer Lett. 2014;342:193-9.

5. Jin Z, Cheng Y, Gu W, Zheng Y, Sato F, Mori Y, Olaru AV, Paun BC, Yang J, Kan T, et al. A multicenter, double-blinded validation study of methylation biomarkers for progression prediction in Barrett's esophagus. Cancer Res. 2009;69:4112-5.

6. Clement G, Braunschweig R, Pasquier N, Bosman FT, Benhattar J. Methylation of APC, TIMP3, and TERT: a new predictive marker to distinguish Barrett's oesophagus patients at risk for malignant transformation. J Pathol. 2006;208:100-7.

7. Salta S, Macedo-Silva C, Miranda-Goncalves V, Lopes N, Gigliano D, Guimaraes R, Farinha M, Sousa O, Henrique R, Jeronimo C. A DNA methylation-based test for esophageal cancer detection. Biomark Res. 2020;8:68.

8. Grady WM, Yu M, Markowitz SD, Chak A. Barrett's esophagus and esophageal adenocarcinoma biomarkers. Cancer Epidemiol Biomark Prev. 2020;29:2486-94.

9. Yu M, Hazelton WD, Luebeck GE, Grady WM. Epigenetic aging: more than just a clock when it comes to cancer. Cancer Res. 2020;80:367-74.

10. Teschendorff AE, Relton CL. Statistical and integrative system-level analysis of DNA methylation data. Nat Rev Genet. 2018;19:129-47.

11. Jaffe $A E$, Irizarry RA. Accounting for cellular heterogeneity is critical in epigenome-wide association studies. Genome Biol. 2014;15:R31.

12. Lutsik P, Slawski M, Gasparoni G, Vedeneev N, Hein M, Walter J. MeDeCom: discovery and quantification of latent components of heterogeneous methylomes. Genome Biol. 2017;18:55.

13. Zheng SC, Beck S, Jaffe AE, Koestler DC, Hansen KD, Houseman AE, Irizarry RA, Teschendorff AE. Correcting for cell-type heterogeneity in epigenome-wide association studies: revisiting previous analyses. Nat Methods. 2017;14:216-7.

14. Jiao $Y$, Widschwendter $M$, Teschendorff AE. A systems-level integrative framework for genome-wide DNA methylation and gene expression data identifies differential gene expression modules under epigenetic control. Bioinformatics. 2014;30:2360-6.

15. Jones A, Teschendorff AE, Li Q, Hayward JD, Kannan A, Mould T, West J, Zikan M, Cibula D, Fiegl H, et al. Role of DNA methylation and epigenetic silencing of HAND2 in endometrial cancer development. PLoS Med. 2013;10:e1001551.

16. Ma X, Sun $P$, Zhang ZY. An integrative framework for protein interaction network and methylation data to discover epigenetic modules. IEEE/ACM Trans Comput Biol Bioinform. 2019;16:1855-66.
17. Ma X, Liu Z, Zhang Z, Huang X, Tang W. Multiple network algorithm for epigenetic modules via the integration of genome-wide DNA methylation and gene expression data. BMC Bioinform. 2017;18:72.

18. Zheng SC, Webster AP, Dong D, Feber A, Graham DG, Sullivan R, Jevons S, Lovat LB, Beck S, Widschwendter M, Teschendorff AE. A novel cell-type deconvolution algorithm reveals substantial contamination by immune cells in saliva, buccal and cervix. Epigenomics. 2018;10:925-40.

19. Cancer Genome Atlas Research N, Asan U, Agency BCC, Brigham, Women's H, Broad I, Brown U, Case Western Reserve U, Dana-Farber Cancer I, Duke U, et al. Integrated genomic characterization of oesophageal carcinoma. Nature. 2017;541:169-75.

20. Theda C, Hwang SH, Czajko A, Loke YJ, Leong P, Craig JM. Quantitation of the cellular content of saliva and buccal swab samples. Sci Rep. 2018;8:6944

21. Wong YT, Tayeb MA, Stone TC, Lovat LB, Teschendorff AE, Iwasiow R, Craig JM. A comparison of epithelial cell content of oral samples estimated using cytology and DNA methylation. Epigenetics. 2021;1-8. https://doi. org/10.1080/15592294.2021.1950977.

22. Nishitani S, Parets SE, Haas BW, Smith AK. DNA methylation analysis from saliva samples for epidemiological studies. Epigenetics. 2018;13:352-62.

23. West J, Beck S, Wang X, Teschendorff AE. An integrative network algorithm identifies age-associated differential methylation interactome hotspots targeting stem-cell differentiation pathways. Sci Rep. 2013;3:1630.

24. Zheng SC, Breeze CE, Beck S, Teschendorff AE. Identification of differentially methylated cell types in epigenome-wide association studies. Nat Methods. 2018;15:1059-66.

25. Owen RP, White MJ, Severson DT, Braden B, Bailey A, Goldin R, Wang LM, Ruiz-Puig C, Maynard ND, Green A, et al. Single cell RNA-seq reveals profound transcriptional similarity between Barrett's oesophagus and oesophageal submucosal glands. Nat Commun. 2018:9:4261.

26. Teschendorff AE, Breeze CE, Zheng SC, Beck S. A comparison of reference-based algorithms for correcting cell-type heterogeneity in Epigenome-Wide Association Studies. BMC Bioinform. 2017;18:105.

27. Singh V, Singh AP, Sharma I, Singh LC, Sharma J, Borthakar BB, Rai AK, Kataki AC, Kapur S, Saxena S. Epigenetic deregulations of Wnt/ beta-catenin and transforming growth factor beta-Smad pathways in esophageal cancer: outcome of DNA methylation. J Cancer Res Ther. 2019;15:192-203.

28. Stairs DB, Bayne LJ, Rhoades B, Vega ME, Waldron TJ, Kalabis J, KleinSzanto A, Lee JS, Katz JP, Diehl JA, et al. Deletion of p120-catenin results in a tumor microenvironment with inflammation and cancer that establishes it as a tumor suppressor gene. Cancer Cell. 2011;19:470-83.

29. Sanchez-Danes A, Blanpain C. Deciphering the cells of origin of squamous cell carcinomas. Nat Rev Cancer. 2018;18:549-61.

30. Lian J, Liu S, Yue Y, Yang Q, Zhang Z, Yang S, Zhang Y. Eomes promotes esophageal carcinoma progression by recruiting Treg cells through the CCL20-CCR6 pathway. Cancer Sci. 2021;112:144-54.

31. Yamamura K, Baba Y, Nakagawa S, Mima K, Miyake K, Nakamura K, Sawayama H, Kinoshita K, Ishimoto T, Iwatsuki M, et al. Human microbiome fusobacterium nucleatum in esophageal cancer tissue is associated with prognosis. Clin Cancer Res. 2016;22:5574-81.

32. Teschendorff AE, Yang Z, Wong A, Pipinikas CP, Jiao Y, Jones A, Anjum S, Hardy R, Salvesen HB, Thirlwell C, et al. Correlation of smoking-associated DNA methylation changes in buccal cells with DNA methylation changes in epithelial cancer. JAMA Oncol. 2015;1:476-85.

33. Issa JP. Aging and epigenetic drift: a vicious cycle. J Clin Invest. 2014;124:24-9.

34. Yang Z, Wong A, Kuh D, Paul DS, Rakyan VK, Leslie RD, Zheng SC, Widschwendter M, Beck S, Teschendorff AE. Correlation of an epigenetic mitotic clock with cancer risk. Genome Biol. 2016;17:205.

35. Teschendorff AE. A comparison of epigenetic mitotic-like clocks for cancer risk prediction. Genome Med. 2020;12:56.

36. Yang $Z$, Jones A, Widschwendter $M$, Teschendorff AE. An integrative pancancer-wide analysis of epigenetic enzymes reveals universal patterns of epigenomic deregulation in cancer. Genome Biol. 2015;16:140.

37. Teschendorff AE, Zheng SC, Feber A, Yang Z, Beck S, Widschwendter M. The multi-omic landscape of transcription factor inactivation in cancer. Genome Med. 2016;8:89.

38. Troyanskaya O, Cantor M, Sherlock G, Brown P, Hastie T, Tibshirani R, Botstein D, Altman RB. Missing value estimation methods for DNA microarrays. Bioinformatics. 2001;17:520-5. 
39. Teschendorff AE, Marabita F, Lechner M, Bartlett T, Tegner J, GomezCabrero D, Beck S. A beta-mixture quantile normalization method for correcting probe design bias in Illumina Infinium 450 k DNA methylation data. Bioinformatics. 2013;29:189-96.

40. Krause L, Nones K, Loffler KA, Nancarrow D, Oey H, Tang YH, Wayte NJ, Patch AM, Patel K, Brosda S, et al. Identification of the CIMP-like subtype and aberrant methylation of members of the chromosomal segregation and spindle assembly pathways in esophageal adenocarcinoma. Carcinogenesis. 2016;37:356-65.

41. Aryee MJ, Jaffe AE, Corrada-Bravo H, Ladd-Acosta C, Feinberg AP, Hansen KD, Irizarry RA. Minfi: a flexible and comprehensive bioconductor package for the analysis of Infinium DNA methylation microarrays. Bioinformatics. 2014;30:1363-9.

42. Kaz AM, Wong CJ, Varadan V, Willis JE, Chak A, Grady WM. Global DNA methylation patterns in Barrett's esophagus, dysplastic Barrett's, and esophageal adenocarcinoma are associated with BMI, gender, and tobacco use. Clin Epigenetics. 2016;8:111.

43. Wettenhall JM, Smyth GK. limmaGUI: a graphical user interface for linear modeling of microarray data. Bioinformatics. 2004;20:3705-6.

44. Lu H, Bhat AA, Peng D, Chen Z, Zhu S, Hong J, Maacha S, Yan J, Robbins DJ, Washington MK, et al. APE1 upregulates MMP-14 via redox-sensitive ARF6-mediated recycling to promote cell invasion of esophageal adenocarcinoma. Cancer Res. 2019;79:4426-38.

45. Butler A, Hoffman P, Smibert P, Papalexi E, Satija R. Integrating single-cell transcriptomic data across different conditions, technologies, and species. Nat Biotechnol. 2018;36:411-20.

46. Cerami EG, Gross BE, Demir E, Rodchenkov I, Babur O, Anwar N, Schultz N, Bader GD, Sander C. Pathway commons, a web resource for biological pathway data. Nucleic Acids Res. 2011;39:D685-690.

47. Rodchenkov I, Babur O, Luna A, Aksoy BA, Wong JV, Fong D, Franz M, Siper MC, Cheung M, Wrana M, et al. Pathway commons 2019 update: integration, analysis and exploration of pathway data. Nucleic Acids Res. 2020;48:D489-97.

48. Ritchie ME, Phipson B, Wu D, Hu Y, Law CW, Shi W, Smyth GK. limma powers differential expression analyses for RNA-sequencing and microarray studies. Nucleic Acids Res. 2015:43:e47.

49. Zhuang J, Widschwendter M, Teschendorff AE. A comparison of feature selection and classification methods in DNA methylation studies using the Illumina Infinium platform. BMC Bioinform. 2012;13:59.

50. Reichardt J, Bornholdt S. Statistical mechanics of community detection. Phys Rev E Stat Nonlinear Soft Matter Phys. 2006;74:016110.

51. Reinius LE, Acevedo N, Joerink M, Pershagen G, Dahlen SE, Greco D, Soderhall C, Scheynius A, Kere J. Differential DNA methylation in purified human blood cells: implications for cell lineage and studies on disease susceptibility. PLoS One. 2012;7:e41361.

52. Iorio F, Knijnenburg TA, Vis DJ, Bignell GR, Menden MP, Schubert M, Aben N, Goncalves E, Barthorpe S, Lightfoot H, et al. A landscape of pharmacogenomic interactions in cancer. Cell. 2016;166:740-54.

53. Smyth GK. Linear models and empirical Bayes methods for assessing differential expression in microarray experiments. Stat Appl Genet Mol Biol. 2004;3:1-25.

\section{Publisher's Note}

Springer Nature remains neutral with regard to jurisdictional claims in published maps and institutional affiliations.
Ready to submit your research? Choose BMC and benefit from:

- fast, convenient online submission

- thorough peer review by experienced researchers in your field

- rapid publication on acceptance

- support for research data, including large and complex data types

- gold Open Access which fosters wider collaboration and increased citations

- maximum visibility for your research: over $100 \mathrm{M}$ website views per year

At BMC, research is always in progress.

Learn more biomedcentral.com/submissions 